\title{
Time Delay Compensation for CDMA Power Control
}

\author{
Fredrik Gunnarsson, Fredrik Gustafsson \\ Division of Communications Systems \\ Department of Electrical Engineering \\ Linköpings universitet, SE-581 83 Linköping, Sweden \\ WWW: http://Www.comsys.isy.liu.se \\ Email: fred@isy.liu.se, fredrik@isy.liu.se
}

August 18, 2000

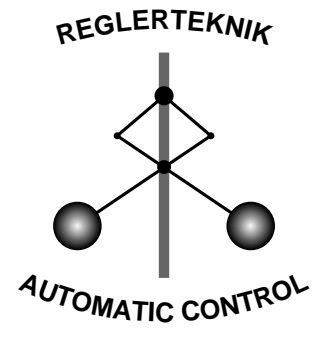

LINKÖPING

Report No.: LiTH-ISY-R-2287

Submitted to GLOBECOM'00, San Francisco, CA, USA

Technical reports from the Communications Systems group in Linköping are available by anonymous ftp at the address ftp.control.isy.liu.se. This report is contained in the file 2287 . pdf. 


\begin{abstract}
Transmission power control is essential in CDMA systems in order to reduce the near-far effect and to optimize the bandwidth utilization, which is critical when variable data rates are used. One remaining problem is oscillations in the output powers due to round-trip delays in the power control loops together with the power up-down command device. The oscillations are naturally quantified using discrete-time describing functions, which are introduced and applied. More importantly, Time Delay Compensation (TDC) is proposed to mitigate the oscillations. It is also formally proven that TDC result in a stable overall system, with power control errors that converges to a defined bounded region. These bounds are tighter, compared to when not employing TDC. Simulations illustrate the oscillations and the significant performance gains using TDC.
\end{abstract}

Keywords: Power control, cellular radio systems, time delays, time delay compensation, discrete-time describing functions, convergence 


\title{
Time Delay Compensation for CDMA Power Control*
}

\author{
Fredrik Gunnarsson, and Fredrik Gustafsson \\ Department of Electrical Engineering \\ Linköpings universitet, SE-581 83 Linköping, SWEDEN \\ Email: fred, fredrik@isy.liu.se \\ Fax: +46 13 282622, Phone: +4613284028
}

\begin{abstract}
Transmission power control is essential in CDMA systems in order to reduce the near-far effect and to optimize the bandwidth utilization, which is critical when variable data rates are used. One remaining problem is oscillations in the output powers due to round-trip delays in the power control loops together with the power up-down command device. The oscillations are naturally quantified using discrete-time describing functions, which are introduced and applied. More importantly, Time Delay Compensation (TDC) is proposed to mitigate the oscillations. It is also formally proven that TDC result in a stable overall system, with power control errors that converges to a defined bounded region. These bounds are tighter, compared to when not employing TDC. Simulations illustrate the oscillations and the significant performance gains using TDC.
\end{abstract}

\section{INTRODUCTION}

In order to utilize the available resources in cellular radio systems efficiently, different radio resource management schemes are needed. One such technique is to control the output powers of the transmitters. In systems based on CDMA, this is particularly important since all terminals are communicating using the same spectrum. Most power control algorithms proposed to date strive to balance the signal-to-interference ratio (SIR) [1].

Fast fading has to be mitigated when possible, and therefore it is desirable to choose a high updating interval of the power control algorithm. The signaling bandwidth is kept low by utilizing only one bit for signaling, where the power is stepwise increased or decreased. Viterbi [2] proposed a scheme where the transmitter power is increased or decreased based on the comparison of received SIR and a threshold. The scheme was further investigated by Ariyavisitakul [3].

Signaling and measuring takes time resulting in time delays in the power control loop, which in turn affects the dynamics of the closed-loop. This has primarily been considered as imperfect power control and Sim et. al. [4] concluded that power control is more sensitive to the delay than to the SIR estimator performance. Chockalingam et. al. [5] indicated, using simulations and analytically approximated second-order statistics,

* This work was supported by the graduate school ECSEL and the Swedish National Board for Industrial and Technical Development (NUTEK), and in cooperation with Ericsson Radio Systems AB, which all are acknowledged. that the performance is degraded when subject to delays in the power control loop. Leibnitz et. al. [6] proposed a Markov chain model to describe the power control dynamics. The horizon of transitions was, however, chosen too short to reveal the presence of oscillations.

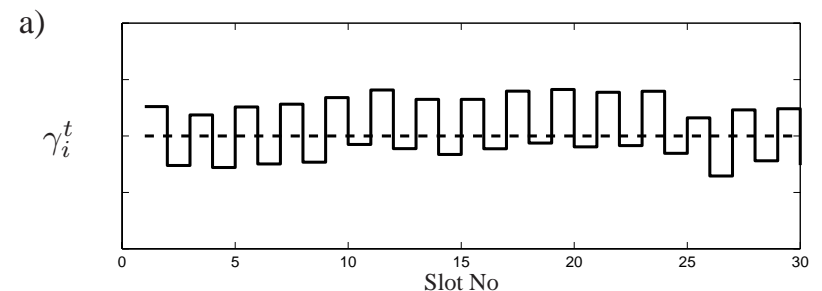

b)

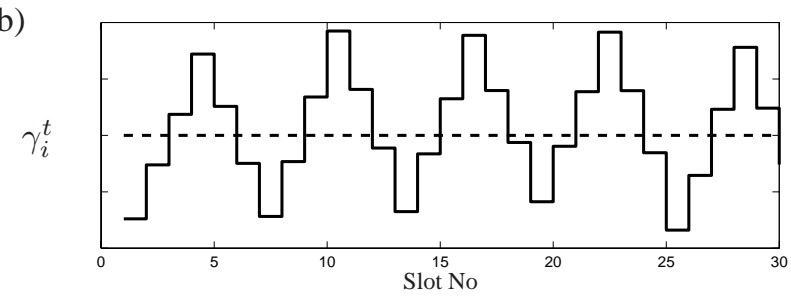

Figure 1: Received SIR in a typical CDMA situation, where the power control commands are delayed by one slot. a) TDC employed, b) no TDC.

The intuitive behavior of a power control algorithm in operation is that the received SIR oscillates up and down around the threshold $\gamma_{i}^{t}(t)$ as in Figure 1 . When subject to delays, however, the amplitude of the oscillations is larger as seen in Figure $1 \mathrm{~b}$. Primarily, time delays results in oscillations in two different ways

1. Delayed reactions to changes in external disturbances.

2. Internal dynamics of the power control loop.

In this paper, time delay compensation (TDC) is proposed to mitigate oscillations due to internal dynamics (second item above). As seen in Figure $1 \mathrm{a}$, which represents the same situation as in Figure $1 b$, but with TDC in operation, the oscillations are significantly reduced. This means that the capacity can be better utilized, which is critical when using variable data rate. 
The rest of the paper is organized as follows. Section [introduces the system models (see also [5, 7]), which are used to describe the power control algorithm in operation. The closedloop system of each power-controlled connection is intuitively depicted in a block diagram capturing the dynamics. Time delay compensation is presented in Section [II] and the dynamics is quantified using discrete-time describing functions in Sections IV and $\mathrm{V}$ A theorem relating to the TDC performance is disclosed in Section VI. The performance improvements using TDC are further illuminated in simulations in Section VII. Finally, Section VIII provide some conclusive remarks.

\section{SySteM MODEL}

Initially it is assumed that each mobile is connected to only one base station, i.e., any soft handover is not considered. This is chosen to keep the notation simple and to emphasize the core ideas. Moreover, for clarity, we consider the uplink. All values will be represented by values in logarithmic scale (e.g., dB or $\mathrm{dBW}$ ). Assume that $m$ active mobile stations transmit using the powers $p_{i}(t), i=1, \ldots, m$. The base stations are seen as several receivers, so that mobile station $i$ is transmitting to receiver $i$. The signal power between mobile station $i$ and receiver $j$ is attenuated by the power gain $g_{i j}(t)(<0)$. Thus the corresponding receiver $i$ will experience a desired signal power

$$
C_{i}(t)=p_{i}(t)+g_{i i}(t)
$$

and an interference plus noise $I_{i}(t)$. The signal-to-interference ratio (SIR) at receiver $i$ is defined by

$$
\gamma_{i}(t)=p_{i}(t)+g_{i i}(t)-I_{i}(t) .
$$

The considered controller is essentially based on a cascade structure, where an inner control loop assigns power levels to meet a target SIR $\gamma_{i}^{t}(t)$ provided by an outer control loop. Since the outer loop is operating more slowly, these targets are considered constant, $\gamma_{i}^{t}(t)=\gamma_{i}^{t}$. The up/down power control device is described by

$$
\begin{aligned}
\text { Receiver : } & e_{i}(t)=\gamma_{i}^{t}-\gamma_{i}(t) \\
s_{i}(t) & =\operatorname{sign}\left(e_{i}(t)\right) \\
\text { Transmitter : } \quad & p_{i}(t+1)=p_{i}(t)+\Delta_{i} s_{i}(t)
\end{aligned}
$$

Only the decisions $s_{i}(t)$ are transmitted over the radio interface, requiring only one bit for signaling. The step size $\delta_{i}$ may be adapted as well. However, such updates are also of slower rate, and the step size is therefore assumed constant. This scheme is sometimes called "CDMA power control". A more descriptive name is Fixed Step Power Control (FSPC), which will be used here. The sign function will be referred to as a relay.

Both measuring and control signaling takes time, and result in time delays in the inner control loop. Since the command signaling is standardized, these delays are known exactly in number of slots. We will adopt the convention that time index $t$ refer to power update instants. As seen in (2), the controller provide a delay of one slot (or sample interval). Moreover, the power control commands may be subject to additional delays of $n$ samples. Typical examples include WCDMA, which operate with an additional delay of one sample (efforts to reduce this delay in some situations are under standardization), and IS-95 which is subject to additional delays of 1-3 samples depending on version. Time delays in the inner control loop are naturally described using the time-shift operator $q$ (note the similarity to the $z$ in the $z$-transform)

$$
q^{n} s_{i}(t)=s_{i}(t+n), q^{-n} s_{i}(t)=s_{i}(t-n) .
$$

Applying the time-shift operator to the power update in the transmitter 2b yields

$$
p_{i}(t)=\frac{\Delta_{i}}{q-1} s_{i}(t)
$$

Furthermore, the observed SIR reflects old transmission powers, due to time delays

$$
\gamma_{i}(t)=p_{i}(t-n)+g_{i i}(t)-I_{i}(t) .
$$

The inner loop can be associated with the block diagram in Figure 2 describing its dynamical behavior.

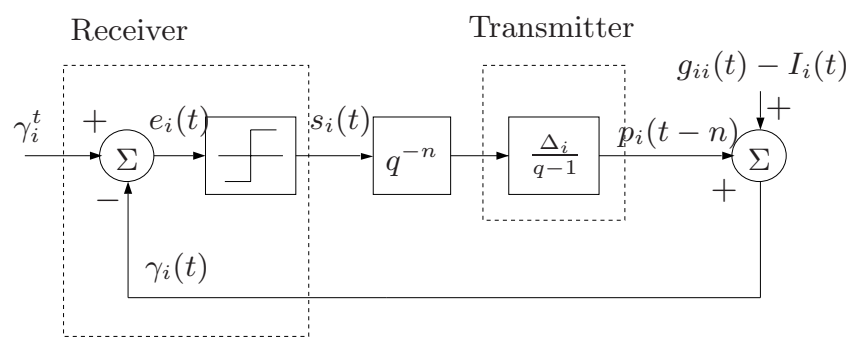

Figure 2: WCDMA inner loop power control, where the power control commands are delayed by $n$ samples.

\section{TIME DELAy COMPENSATION}

Time delays affect the stability and performance of any controlled system. Essentially, the core problem is that the measurements do not reflect the most recent power updates. However, these are known to the algorithm, and can be compensated for. In this section we discuss such a compensation strategy with respect to the FSPC algorithm in (2). The more general concept of time delay compensation applied to a general power control algorithm with, as well as to the most popular algorithms, is further discussed by Gunnarsson in [8, 9]. Loop filters and nonlinear components are also taken into consideration.

The main idea is to in the receiver monitor the powers to be used by the transmitter. These are then used to form adjusted SIR measurements, $\breve{\gamma}_{i}(t)$. Time delay compensation (TDC) is formalized in the following algorithm, where limited dynamic range also is considered. 


\section{Algorithm 1 (FSPC with TDC)}

i) Adjust measurements: $\breve{\gamma}_{i}(t)=\gamma_{i}(t)+\breve{p}_{i}(t)-\breve{p}_{i}(t-n)$.

ii) Issue power control command: $s_{i}(t)=\operatorname{sign}\left(\gamma_{i}^{t}-\breve{\gamma}_{i}(t)\right)$.

iii) Monitor output powers to be used: $\breve{p}_{i}(t+1)=\max \left(p_{\min }, \min \left(p_{\max }, \breve{p}_{i}(t)+\beta_{i} s_{i}(t)\right)\right)$.

Using geometric series, it is easy to verify that

$$
\breve{p}_{i}(t)-\breve{p}_{i}(t-n)=\beta \sum_{j=1}^{n} s_{i}(t-j) .
$$

Algorithm 1 can thus be rewritten as

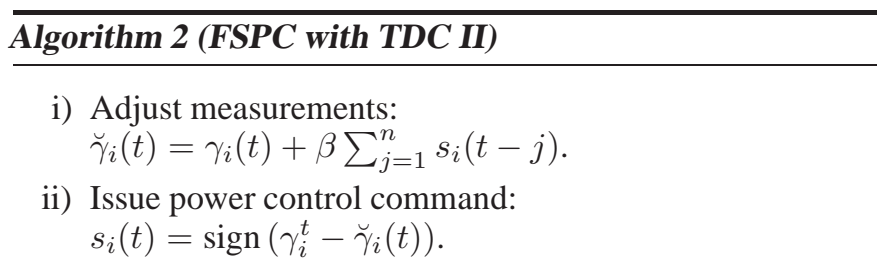

Hence, the cost of implementing TDC compared to employing FSPC in (2), is only a few (in the typical case $n=1$ only one) addition(s). Essentially, the effect of TDC is that the internal additional round-trip delays ( $n$ samples) are all cancelled. However, the controller still encompasses delayed reactions to changes in target SIR and other external disturbances.

Some specific situations require special attention. For example, the uplink when in soft handover. While in operation, a base station is unaware of whether an issued power control command is applied or not, since the command from another base station may have been prioritized. Therefore, TDC should be disabled in the uplink while in soft handover. The applicability in the downlink when in soft or softer handover is depending on the combining strategy in the receiver.

Another consideration is that the power control bits $s_{i}(t)$ might be subject to bit errors. A simulation study of these effects is provided in SectionVII

\section{Discrete-Time Describing Functions}

Static nonlinearities, such as the relay (or sign function) in the FSPC algorithm (2), in closed control loops may result in persistent oscillations. These oscillations can be approximated using discrete-time describing functions [9, 10]. In this section we briefly review the technique,

Basically, we are focusing on loops that consist of a linear part with transfer function $G(q)$ and a static nonlinearity described by the function $f(\cdot)$ resulting in a loop as in Figure 3 Note that we have assumed a zero input to the loop. Nonzero inputs are studied in Section $\mathrm{V}$. The underlying assumption is the

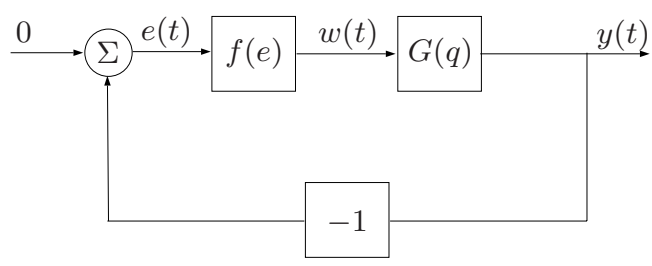

Figure 3: Block diagram of a nonlinear system, separated into one linear and one nonlinear component.

existence of an oscillation in the error signal $e(t)$, and then try to verify this assumption. We proceed by making the $N$ periodic hypothesis

$$
e(t)=E \sin \left(\Omega_{e}\left(t+\delta_{e}\right)\right)=E \sin \left(\frac{2 \pi}{N}\left(t+\delta_{e}\right)\right),
$$

where the unknown time shift $\delta_{e} \in[0,1[$ is motivated by the fact that discontinuous nonlinearities may result in time shifts. For example, consider a relay and $N$ even.

$$
w(t)=\operatorname{sign}\left(E \sin \left(\frac{2 \pi}{N}\left(t+\delta_{e}\right)\right)\right)=\operatorname{sign}\left(E \sin \left(\frac{2 \pi}{N}(t)\right)\right) .
$$

Note that a time shift of an entire sample is the same as a time delay and should therefore be a part of of the linear transfer function $G(q)$.

An underlying assumption is that the linear part $G(q)$ attenuates the harmonics much more than the fundamental frequency in the loop. More formally, we assume

\section{Assumption 1}

The linear part $G(q)$ in Figure 3 attenuates the harmonics much more than the fundamental frequency $\Omega_{e}$. More formally, we assume that

$$
\left.\frac{\left|G\left(e^{i k \Omega_{e}}\right)\right|}{\left|G\left(e^{i \Omega_{e}}\right)\right|}\right|_{\Omega_{e}=\frac{2 \pi}{N_{0}}}<<1, \quad k=2, \ldots, \frac{N_{0}}{2}
$$

The discrete-time describing functions analysis is summarized in the following algorithm. For details, see [9,11]. Preliminary work is found in [7]

\section{Algorithm 3 (Discrete-Time Describing Functions Analysis)}

Consider the situation in Figure 3 , where the loop is separated in a linear $(G(q))$ and a nonlinear $(f(\cdot))$ part. Then the oscillation in the error signal $e(t)$ is approximated by the procedure 
1. Determine the discrete-time describing function of the nonlinearity as

$Y_{f}\left(E, N, \delta_{e}\right)=\frac{2 i}{N E} \sum_{t=0}^{N-1} f\left(E \sin \left(\Omega_{e}\left(t+\delta_{e}\right)\right)\right) e^{-i\left(\Omega_{e}\left(t+\delta_{e}\right)\right)}$

where $\Omega_{e}=\frac{2 \pi}{N}$ and $\delta_{e} \in[0,1[$.

2. Compute $\left.G(q)\right|_{q=e^{2 \pi i / N}}$.

3. Solve the following equation for $E, N$ and $\delta_{e}$.

$$
Y_{f}\left(E, N, \delta_{e}\right) G\left(e^{i \frac{2 \pi}{N}}\right)=-1
$$

If one solution $\left(E, N, \delta_{e}\right)$ exists, then the oscillation is approximated by

$$
e(t)=E \sin \left(\frac{2 \pi}{N}\left(t+\delta_{e}\right)\right)
$$

If several solutions exist, then several modes of oscillation are possible.

4. Investigate the correctness of Assumption 1. If it does not hold, the estimated periods are still informative, but alternative waveforms may be discussed.

The technique is applied to power control in the following section.

\section{DYNAMICAL BEHAVIOR OF CDMA POWER CONTROL}

In this particular case with integral action in the controller, the oscillations, if any, will oscillate around target SIR. Therefore, we can without loss of generality assume that $\gamma_{i}^{t}=0 \mathrm{~dB}$ and $N$ is even. For a more rigorous motivation, see [9].

\section{A. Discrete-time Describing Function of a Relay}

An ideal relay or sign function is defined by

$$
f(e)=\left\{\begin{array}{rr}
1, & e \geq 0 \\
-1, & e<0
\end{array}\right.
$$

The corresponding describing function is obtained by considering the first step in Algorithm 3

$$
\begin{aligned}
Y_{f}\left(E, N, \delta_{e}\right) & =\frac{2 i}{N E} \sum_{t=0}^{N-1} f\left(E \sin \left(\frac{2 \pi}{N}\left(t+\delta_{e}\right)\right)\right) e^{-i\left(\frac{2 \pi}{N}\left(t+\delta_{e}\right)\right)}= \\
& =\frac{4}{N E \sin \left(\frac{\pi}{N}\right)} e^{i\left(\frac{\pi}{N}-\delta_{e} \frac{2 \pi}{N}\right)}
\end{aligned}
$$

\section{B. Linear Part in the Power Control Loop}

From Figure 2 we conclude that the linear part $G(q)$ compiles to

$$
G(q)=\frac{\Delta_{i}}{q^{n}(q-1)} .
$$

On the unit circle, the following holds

$$
\left.G(q)\right|_{q=e^{2 \pi i / N}}=\frac{\Delta_{i}}{2 \sin (\pi / N)} e^{-i\left(\frac{\pi}{2}+\frac{\pi}{N}+n \frac{2 \pi}{N}\right)}
$$

\section{Predicting Oscillations}

According to Algorithm 3, the oscillations (if any) are characterized by the solutions to the equation

$$
Y_{f}\left(E, N, \delta_{e}\right) G\left(e^{i \frac{2 \pi}{N}}\right)=-1
$$

Separating the magnitude and phase yield two equations

$$
\begin{gathered}
E=\frac{2 \beta_{i}}{N \sin ^{2} \frac{\pi}{N}} \\
\frac{\pi}{2}+\frac{2 \pi}{N}\left(\delta_{e}+n\right)=\pi+2 \pi \nu, \quad \nu \in \mathbb{Z}, \delta_{e} \in[0,1[
\end{gathered}
$$

The phase equation allow several solutions. Since $N$ is an even integer, some reasoning yields that the even $N$ :s satisfying $10 \mathrm{~b}$ are the integers given by

$$
N=\frac{4\left(\delta_{e}+n\right)}{1+4 \nu}, \quad \nu=0,1,2, \ldots, \delta_{e} \in\left\{0, \frac{1}{2}\right\} .
$$

Note that when TDC is employed, $N$ is always equal to 2 , since it cancels the internal delays. For each $N$, the corresponding amplitude is computed using (10a). Finally, we have to verify the correctness of Assumption 1. If it does not hold, the estimated periods are still informative, as pointed out in [9, 11]. With the step-wise updates of the relay, an intuitive alternate waveform is a triangular wave. Such a waveform of period $N$ has the amplitude

$$
E^{\prime}=\frac{N \beta_{i}}{4}
$$

\section{Analysis of a Typical CDMA Case}

In a typical WCDMA or IS-95 situation, $n=1$. Using (11), we predict oscillations of period $N=6$ and $N=4$ (only $\nu=0$ is possible.), using 111. The analysis is summarized in Table 1 approximating the amplitude using both (10a) and (12). Computations reveals that Assumption 1 does not hold in this case. Therefore, $E_{0}^{\prime}$ provide better amplitude estimates. We note that the simulated behavior in Figure $1 \mathrm{~b}$ is well described by the first mode. The current oscillation mode depend on the unknown time shift $\delta_{e}$. A speculative proposition is that the contribution from nonzero inputs might be neglected, but they can be seen as stimulating mode switching, between the in this case two modes. 


\begin{tabular}{|c|l|l|l|}
\hline Oscillation mode & $E_{0}$ & $E_{0}^{\prime}$ & $\delta_{e}$ \\
\hline \hline$N_{0}=4$ & $\beta_{i}$ & $\beta_{i}$ & 0 \\
$N_{0}=6$ & $1.33 \beta_{i}$ & $1.5 \beta_{i}$ & 0.5 \\
\hline
\end{tabular}

Table 1: Predicted oscillation modes in the typical CDMA situation.

\section{CONVERGENCE}

The benefits using TDC result in smaller bounds on the deviations from SIR targets, as disclosed in the following theorem. Note, however, that a tighter bound not necessarily imply a smaller error.

\section{Theorem 2}

Assume that the power control problem is feasible. Then there exists a $t_{\text {lim }} \geq 0$ such that the error when using FSPC with and without TDC is bounded by

$$
\begin{aligned}
& \text { FSPC, no TDC: } \quad\left|\gamma_{i}^{t}-\gamma_{i}(t)\right| \leq \Delta(2 n+2) \\
& \text { FSPC, with TDC: } \quad\left|\gamma_{i}^{t}-\gamma_{i}(t)\right| \leq \Delta(n+2)
\end{aligned}
$$

for $t \geq t_{l i m}$.

Proof The bound of FSPC without TDC was proved in [12]. For the latter see Gunnarsson [9].

\section{Simulations}

The effect of command bit errors on the TDC performance might be critical. This has been studied in network simulations using a simulation environment of WCDMA system (see [13] for simulator details), using $n=1$. We used the standard deviation of the error $e_{i}(t)=\gamma_{i}^{t}-\gamma_{i}(t)$ to compare the performance with and without TDC. The situation of a specific user is compared in Figure 1. The result with respect to different command bit error rates $p_{C B E R}$ in Figure 4indicate that TDC is still beneficial for $p_{C B E R} \leq 0.1$.

\section{CONCLUSIONS}

Time delays in the power control loop together with the typical CDMA power control algorithm result in an oscillative behavior. This dynamical behavior is predicted using discrete-time describing function, which are introduced and applied. The oscillations comprise several modes of oscillation, and mode switching may be stimulated by external signals. To reduce the oscillations, time delay compensation (TDC) is introduced. The result is less oscillative behavior, which is observed both in WCDMA simulations and in describing functions analysis. Furthermore, it can be formally proven that CDMA power control with TDC yield a stable overall system and the SIR error converges to a defined region. The bound on this convergence region is tighter when using TDC than when not.

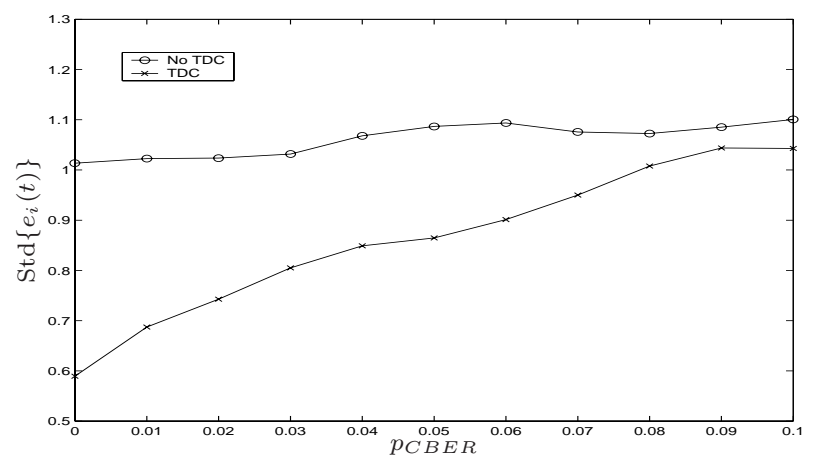

Figure 4: Standard deviation of the received SIR error in a typical WCDMA situation, where the power control commands are delayed by one slot. a) TDC employed, b) no TDC.

\section{REFERENCES}

[1] J.M. Aein. Power balancing in systems employing frequency reuse. COMSAT Technical Review, 3(2), 1973.

[2] A.J. Viterbi, A.M. Viterbi, and E. Zehavi. Performance of power controlled wideband terrestrial digital communication. IEEE Transactions on Communications, 41(4), 1993.

[3] S. Ariyavisitakul and L.F. Chang. Signal and interference statistics of a CDMA system with feedback power control. IEEE Transactions on Communications, 41(11), 1993.

[4] M.L. Sim, E. Gunawan, C.B. Soh, and B.H. Soong. Characteristics of closed loop power control algorithms for a cellular DS/CDMA system. IEE Proceedings - Communications, 147(5), October 1998.

[5] A. Chockalingam, P. Dietrich, L. B. Milstein, and R.R. Rao. Performance of closed-loop power control in DS-CDMA cellular systems. IEEE Transactions on Vehicular Technology, 47(3), August 1998.

[6] K. Leibnitz, P. Tran-Gia, and J.E. Miller. Analysis of the dynamics of CDMA reverse link power control. In Proc. IEEE Global Telecommunications Conference, Sydney, Australia, November 1998.

[7] F. Gunnarsson, J. Blom, and F. Gustafsson. Power control in cellular systems subject to constraints and time delays. In Proc. IEEE Global Telecommunications Conference, Sydney, Australia, November 1998.

[8] F. Gunnarsson and F. Gustafsson. Power control with time delay compensation. In Proc. IEEE Vehicular Technology Conference, Boston, MA, USA, Sep 2000.

[9] F. Gunnarsson. Power Control in Cellular Radio System: Analysis, Design and Estimation. $\mathrm{PhD}$ thesis, Linköpings universitet, Linköping, Sweden, April 2000.

[10] M. de la Sen, A. Peña, and J. Esnaola. Detection of limit cycles in discrete systems with backlash and resolution by using a discretizationoriented describing function. In Proc. IEEE Conference on Decision and Control, San Antonio, TX, USA, December 1993.

[11] F. Gunnarsson, F. Gustafsson, and J. Blom. Dynamical effects of time delays and time delay compensation in power controlled WCDMA. Submitted to IEEE Journal on Selected Areas in Communications, WCDMA Special Issue., 2000.

[12] J.D. Herdtner and E.K.P. Chong. Analysis of a class of distributed asynchronous power control algorithms for cellular wireless systems. To appear in IEEE Journal on Selected Areas in Communications, 2000.

[13] B. Engström and M. Ericsson. WCDMA system level performance with fast fading and non-ideal power control. In Proc. IEEE Vehicular Technology Conference, September 1999. 